\title{
A Cage-monoterpene Indole Alkaloid from Alstonia scholaris
}

\author{
Xiang-Hai Cai, Qin-Gang Tan, Ya-Ping Liu, Tao Feng, Zhi-Zhi Du, Wei-Qi Li, \\ Xiao-Dong Luo*
}

\begin{abstract}
Affiliation
State Key Laboratory of Phytochemistry and Plant Resources in West China Kunming Institute of Botany, Chinese Academy of Sciences, Kunming, People’s Republic of China
\end{abstract}

\section{Correspondence}

Prof. Dr. Xiao-Dong Luo, State Key Laboratory of Phytochemistry and Plant Resources in West China, Kunming Institute of Botany, Chinese Academy of Sciences, Heilongtan, Kunming 650204, People’s Republic of China. E-mail: xdluo@mail.kib.ac.cn. Phone: +86 871 5223188; Fax: +86 8715150227 


\section{Table of Contents}

Figure 1. ${ }^{1} \mathrm{H}$ NMR spectrum of scholarisine A. (1)

Figure 2. ${ }^{13} \mathrm{C}$ NMR spectrum of scholarisine A (1)

Figure 3. ${ }^{1} \mathrm{H}-{ }^{1} \mathrm{H}$ COSY spectrum of scholarisine A (1)

Figure 4. HSQC spectrum of scholarisine A. (1)

Figure 5. HMBC spectrum of scholarisine A (1)

Figure 6. ROESY spectrum of scholarisine A (1)

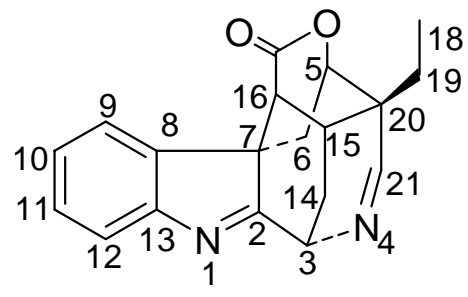

scholarisine A (1) 
Figure 1. ${ }^{1} \mathrm{H}$ NMR spectrum of scholarisine A (1)

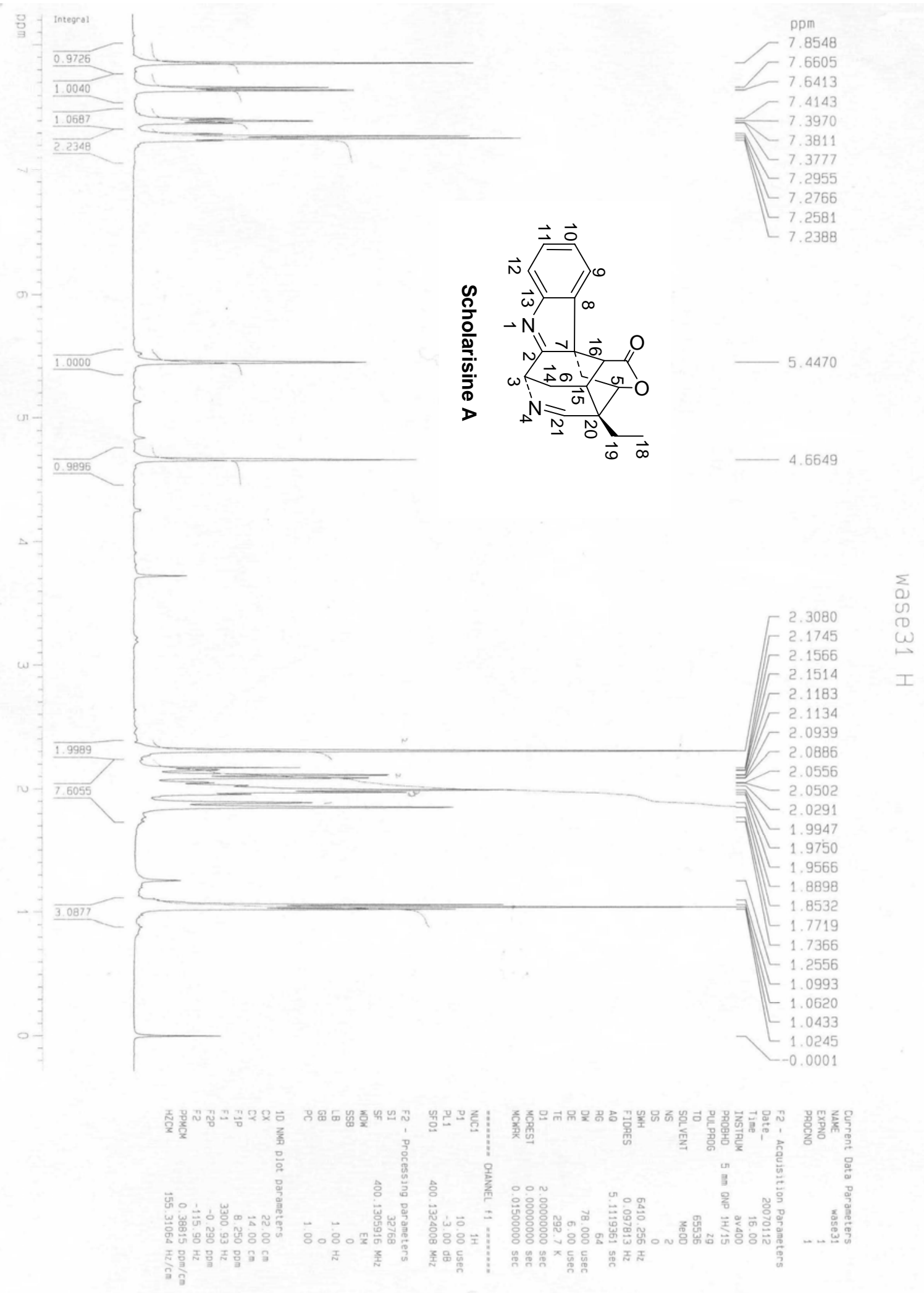


Figure 2. ${ }^{13} \mathrm{C}$ NMR spectrum of scholarisine A (1)
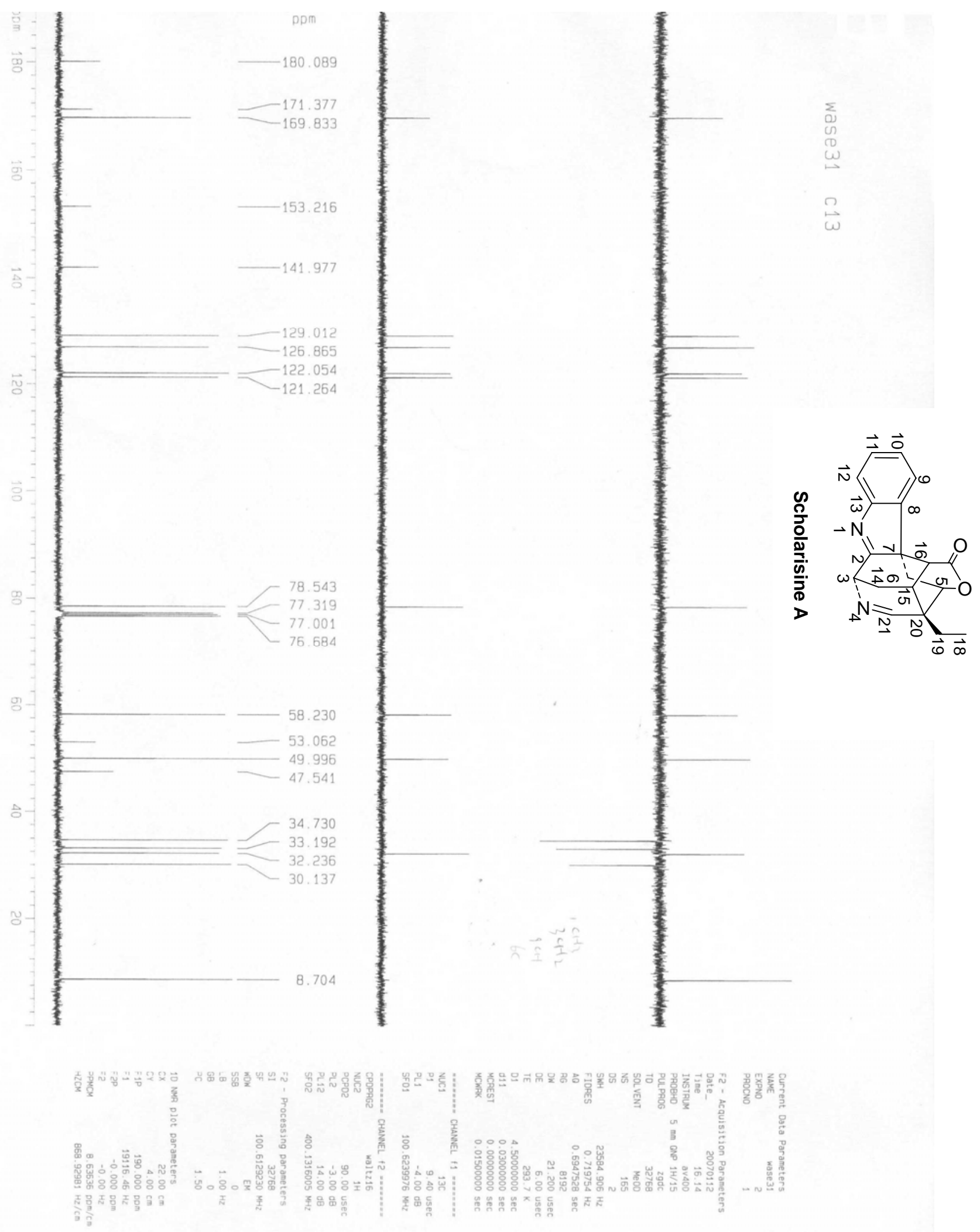
Figure 3. ${ }^{1} \mathrm{H}-{ }^{1} \mathrm{H}$ COSY spectrum of scholarisine A (1)
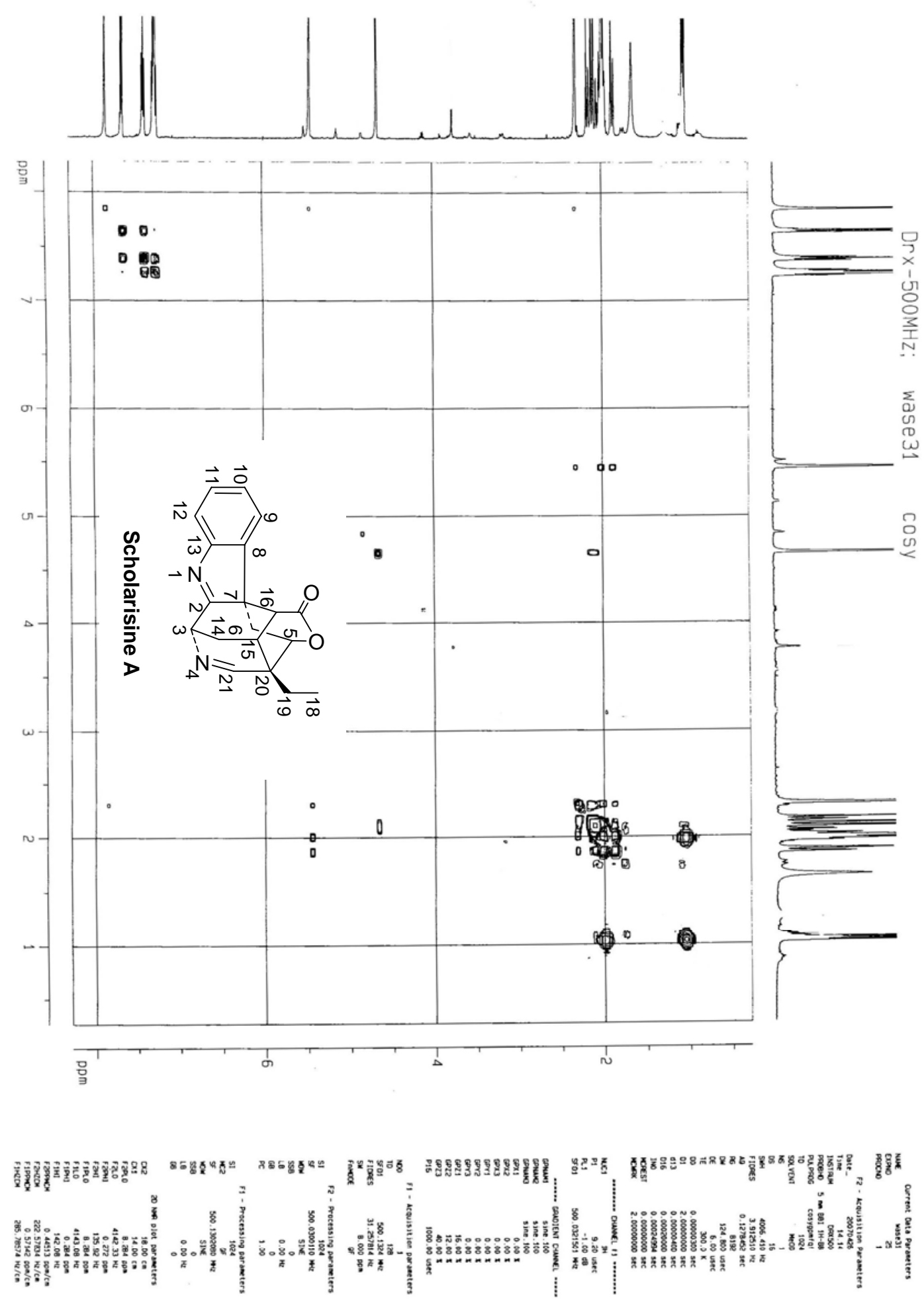
Figure 4. HSQC spectrum of scholarisine A (1)
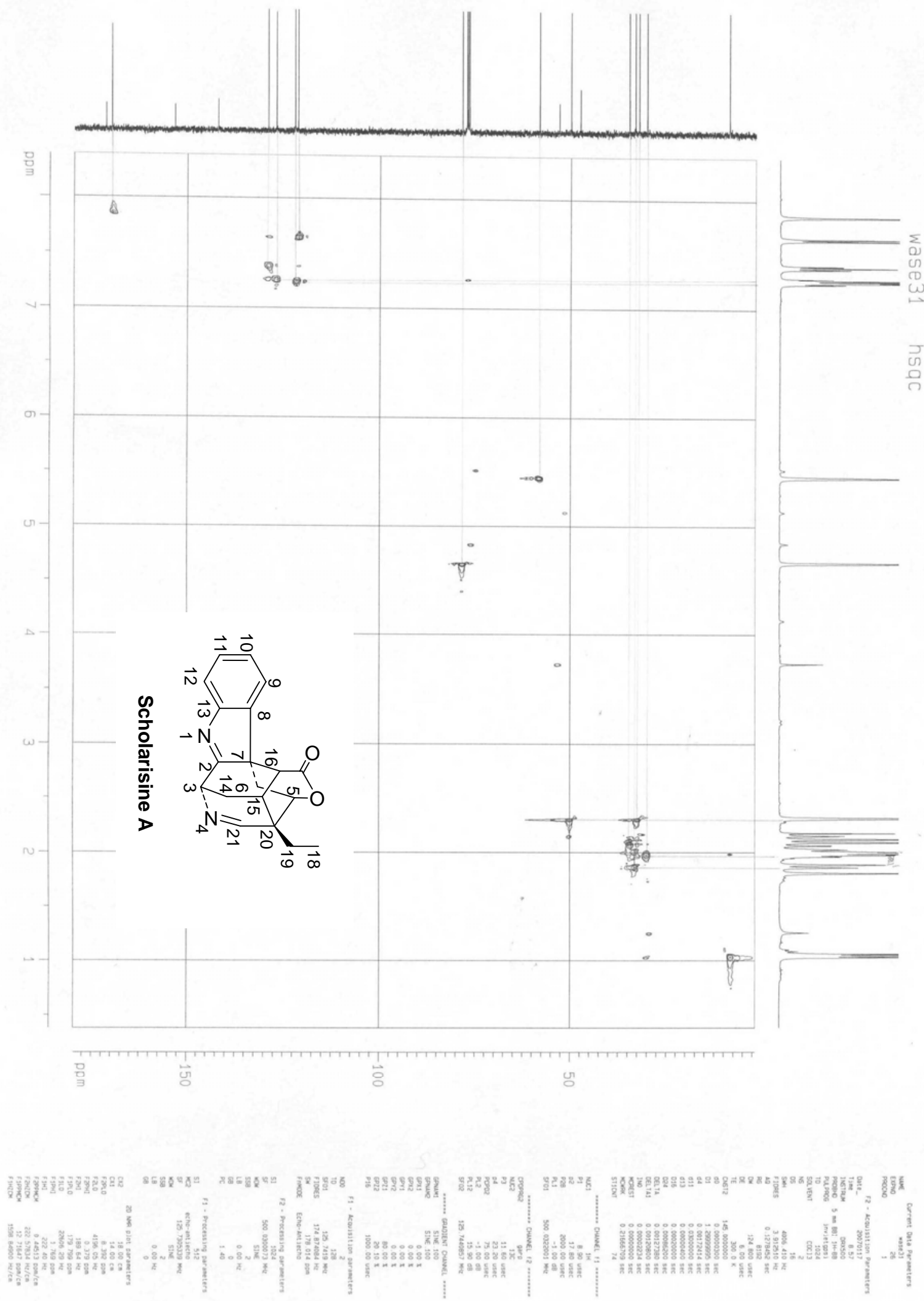
Figure 5. HMBC spectrum of scholarisine A (1)

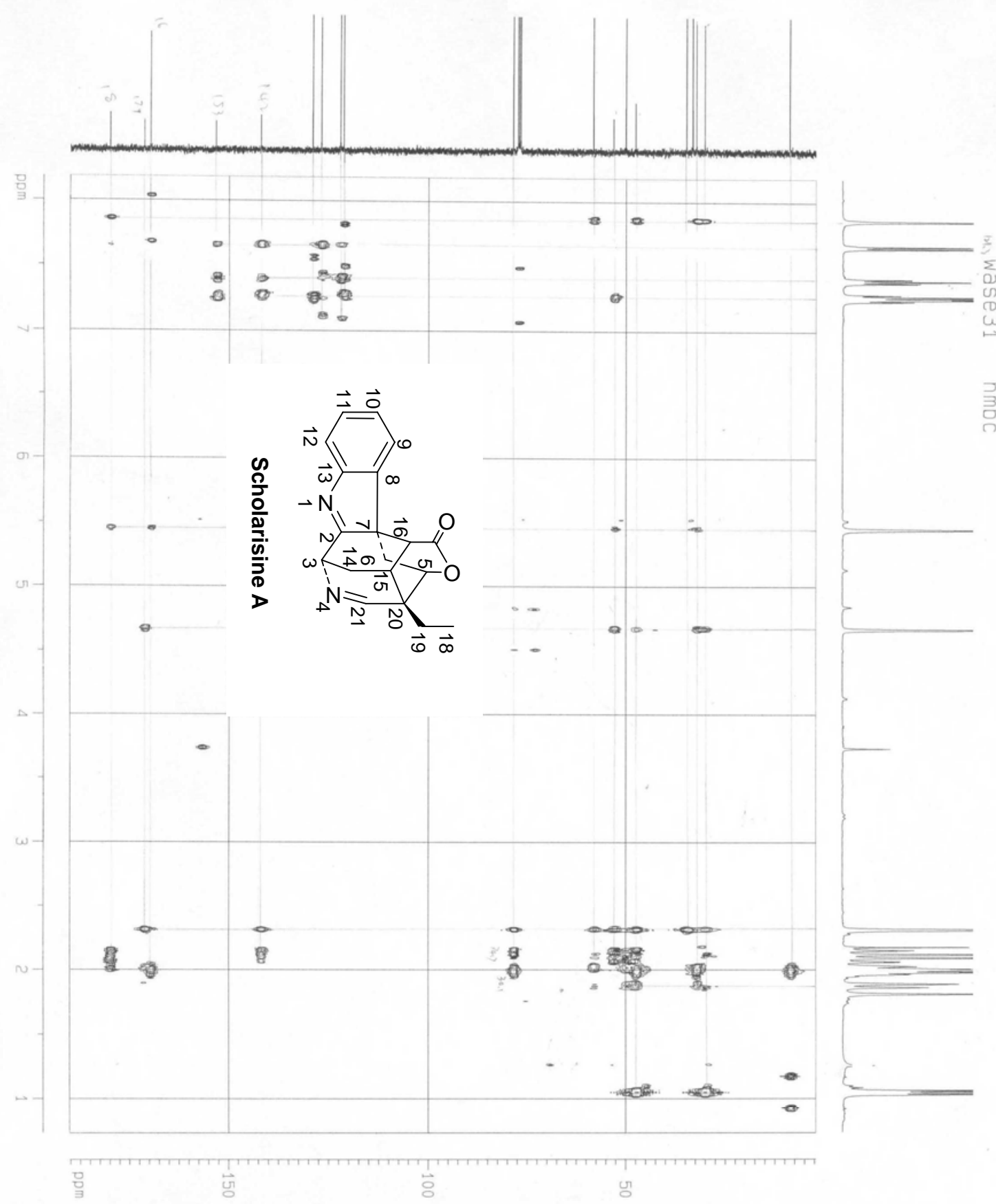

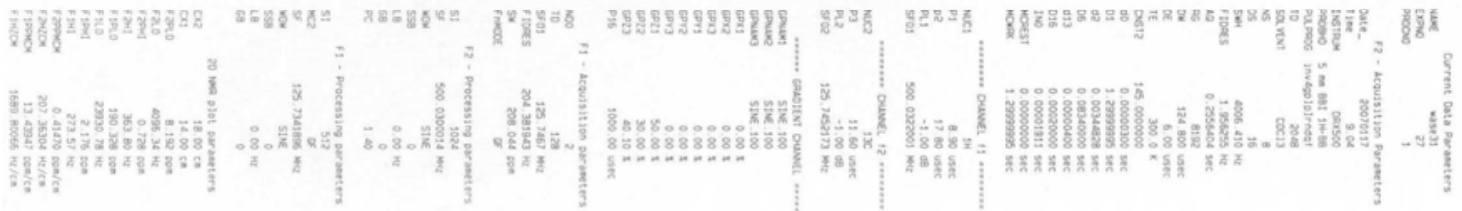


Figure 6. ROESY spectrum of scholarisine A (1)
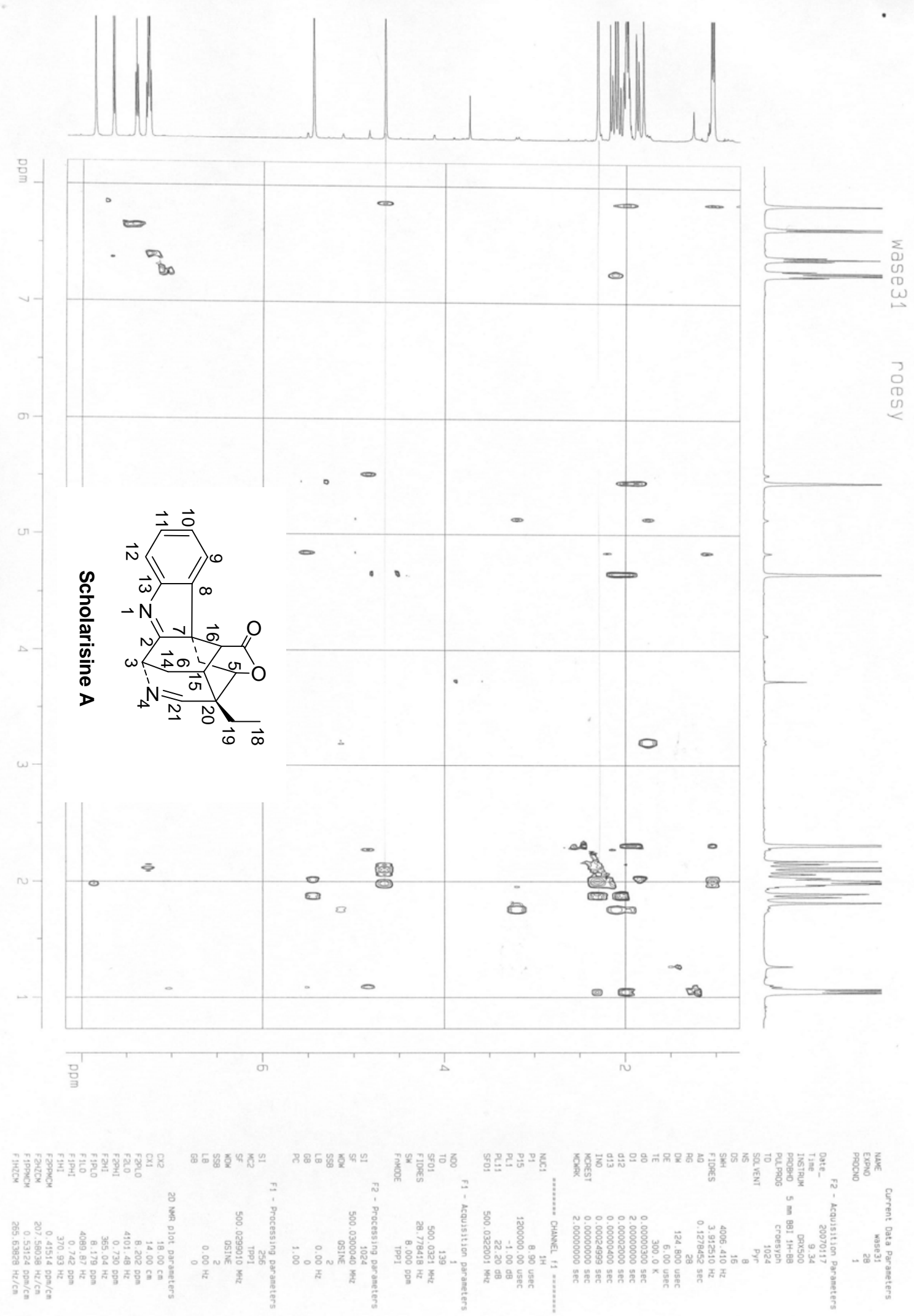